\title{
RESEARCH
}

Open Access

\section{STEAP4 expression in CNS resident cells promotes Th17 cell-induced autoimmune encephalomyelitis}

\author{
Junjie Zhao ${ }^{1}$, Yun Liao' ${ }^{1}$ William Miller-Little ${ }^{1}$, Jianxing Xiao ${ }^{1}$, Caini Liu', Xiaoxia Li ${ }^{1}$, Xiao Li $^{2^{*}}$ and Zizhen Kang ${ }^{1,3^{*}}$ (D)
}

\begin{abstract}
Background: Multiple sclerosis (MS) is a debilitating neurological disease caused by autoimmune destruction of the myelin sheath. Experimental autoimmune encephalomyelitis (EAE) is a widely used animal model for the pathogenesis of MS. We and others have previously demonstrated that IL-17 is critical for the pathogenesis of EAE. The concentration of IL-17 is significantly higher in the sera of MS patients than in healthy controls and correlates with disease activity. Moreover, anti-IL-17 neutralizing antibody demonstrated promising efficacy in a phase II trial in MS patients, further substantiating a key pathogenic role for IL-17 in MS. While Th17 and IL-17 are emerging as a bona fide drivers for neuroinflammation, it remains unclear what effector molecule executes the inflammatory tissue destruction in Th17-driven EAE.

Methods: By microarray analysis, we found STEAP4 is a downstream molecule of IL-17 signaling in EAE. We then used STEAP4 global knockout mice and STEAP4 conditional knockout mice to test its role in the pathogenesis of EAE.

Results: Here, we report that the metalloreductase, STEAP4, is a key effector molecule that participates and contributes to the pathogenesis of Th17-mediated neuroinflammation in experimental autoimmune encephalomyelitis. STEAP4 knockout mice displayed delayed onset and reduced severity of EAE induced by active immunization. The reduced disease phenotype was not due to any impact of STEAP4 deficiency on myelin reactive T cells. In contrast, STEAP4 knockout mice were resistant to passively induced EAE, pointing to a role for STEAP4 in the effector stage of EAE. Notably, STEAP4 was only induced the spinal cord of EAE mice that received Th17 cells but not Th1 cells. Consistently, STEAP4 deficiency protected from only Th17 but not Th1-induced EAE. Finally, using Nestin-Cre STEAP4 $4^{\mathrm{fl} / \mathrm{fl}}$ mice, we showed that ablation of STEAP4 expression in the resident cells in the central nervous system attenuated disease severity in both active immunization and passive Th17 transfer-induced EAE.
\end{abstract}

Conclusion: In this study, we identified STEAP4 as a Th17-specific effector molecule that participates and contributes to the pathogenesis of neuroinflammation, thus potentially provide a novel target for MS therapy.

Keywords: Steap4, Th17, Interleukin-17, EAE, Multiple sclerosis

\footnotetext{
*Correspondence: xiao.li9@case.edu; zizhen-kang@uiowa.edu

${ }^{2}$ The Center for RNA Science and Therapeutics, Case Western Reserve University, Cleveland, OH, USA

'Department of Inflammation and Immunity, Lerner Research Institute, Cleveland, OH, USA

Full list of author information is available at the end of the article
}

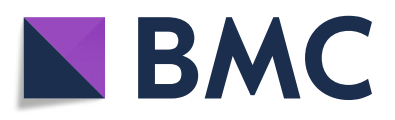

(- The Author(s). 2021 Open Access This article is licensed under a Creative Commons Attribution 4.0 International License, which permits use, sharing, adaptation, distribution and reproduction in any medium or format, as long as you give appropriate credit to the original author(s) and the source, provide a link to the Creative Commons licence, and indicate if changes were made. The images or other third party material in this article are included in the article's Creative Commons licence, unless indicated otherwise in a credit line to the material. If material is not included in the article's Creative Commons licence and your intended use is not permitted by statutory regulation or exceeds the permitted use, you will need to obtain permission directly from the copyright holder. To view a copy of this licence, visit http://creativecommons.org/licenses/by/4.0/. The Creative Commons Public Domain Dedication waiver (http://creativecommons.org/publicdomain/zero/1.0/) applies to the data made available in this article, unless otherwise stated in a credit line to the data. 


\section{Introduction}

Experimental autoimmune encephalomyelitis (EAE) is a widely used animal model for the pathogenesis of multiple sclerosis (MS), a debilitating neurological disease caused by autoimmune destruction of the myelin sheath [1-3]. While $\mathrm{T}$ helper cells were shown to drive the neuroinflammation in the EAE model in 1980s, significant progresses in elucidating the pathogenic mechanism were made predominantly in the past two decades [4-6]. The discovery of Th17, a unique $\mathrm{T}$ helper cell subset that produces IL-17, led to a major realignment of our understanding of the immunopathogenic process [7-9]. While myelin-reactive Th1 and Th17 cells can both mediate encephalomyelitis when they are transferred into sublethally irradiated mice, abrogation of Th17 cell differentiation renders mice resistant to the induction of EAE by active immunization $[9,10]$, highlighting a more fundamental role for Th17 lineage cells in a normal course of autoimmune pathogenesis.

As the signature cytokine of Th17 cells, IL-17 subsequently became the focus of study as the culprit cytokine that drives neuroinflammation in EAE [11-13]. Prevailing evidence indicate that $\mathrm{IL}-17$ is required for the pathogenesis of EAE in mice models. Mice deficient in IL-17 or IL-17 receptor are protected from active immunization-induced EAE [11-13]. IL-17 binds a heterodimer receptor complex, which then transduces the signal through an adaptor molecule, Act1 [14, 15]. Taking advantage of the critical role for Act1 in IL-17 signaling, we have previously completed and reported a series of studies examining the cell-type specific role of IL-17 signaling in EAE pathogenesis. These efforts have identified the resident cells in the central nervous systems (CNS), especially the NG2+ oligodendrocyte progenitor cells, as the primary target of IL-17 and critical drivers for Th17-mediated EAE [16, 17]. In agreement with the findings from the EAE model, correlative studies using patient specimen have strongly implicated IL-17 in the pathogenesis of MS. The concentration of IL-17 is significantly higher in the sera of MS patients than in healthy controls and correlates with disease activity. Moreover, anti-IL-17 neutralizing antibody demonstrated promising efficacy in a phase II trial in MS patients, further substantiating a key pathogenic role for IL-17 in MS [18].

While Th17 and IL-17 are emerging as a bona fide drivers for neuroinflammation, it remains unclear what effector molecule executes the inflammatory tissue destruction in Th17-driven EAE. Addressing this question would be crucial for our understanding of the underlying process, as myelin-reactive Th1 cells are equally potent at mediating demyelination, yet Th1 lineage cells are dispensable during active EAE [9]. To identify effector molecules that mediate Th17-driven neuroinflammation, we performed transcriptomic profiling of spinal cord tissue from EAE mice that received myelin-reactive Th17 or Th1 cells. Differential gene expression analysis identified STEAP4, as a target gene that was highly induced in Th17- but not Th1-induced EAE mice.

STEAP4 (six-transmembrane epithelial antigen of the prostate) encodes a transmembrane protein that consist of an N-terminal cytoplasmic oxidoreductase domain (OxRD) and a six-helical transmembrane domain (TMD). STEAP family proteins were originally cloned from prostate cancer cells [19] and were later found to exhibit metalloreductase activity that catalyzes iron(III) and copper (II) reduction using intracellular NADPH [20-23]. Elevated expression of STEAP4 has been linked to pathogenesis of intestinal inflammation [24], cancer $[25,26]$, and metabolic diseases [27]. In this study, we sought to investigate the role of STEAP4 as an effector molecule in the pathogenesis of Th17-driven EAE.

\section{Materials and methods \\ Mice}

All mice used in the experiment were housed under specific pathogen-free conditions. STEAP4-deficient mice and STEAP4 ${ }^{\text {floxed/floxed }}$ mice in the C57BL/6J background were generated as described [25, 28]. B6.Cg$\mathrm{Tg}(\mathrm{Nes}-\mathrm{Cre}) 1 \mathrm{Kln} / \mathrm{J}$ (NesCre transgenic ) [29, 30], C57BL/ 6J were purchased from Jackson laboratory. For all experiments, mice were 6-12 weeks old, age-matched littermates between experimental groups. Experimental protocols were approved by the Institutional Animal Care and Use Committee of Cleveland Clinic.

\section{Transcriptomic profiling of EAE spinal cord}

The transcriptomic profiling experiment that led to the identification of STEAP4 as a target gene in Th17induced EAE was first described in our previous study [31]. Briefly, five mice in each group were left untreated (naive group) or transferred with MOG-reactive Th1 or Th17 cells. At peak of disease, the mice were killed, and spinal cords were harvested for mRNA extraction, followed by microarray analysis. The samples was hybridized on an Affymetrix GeneChip HT-MG-430PM-96 (Affymetrix). Three independent biological replicates were analyzed in each experiment, which yielded consistent results. The dataset is deposited the Gene Expression Omnibus under accession code GSE97035.

\section{Induction and assessment of EAE}

Active EAE and passive EAE was induced and assessed as previously described [14, 17]. In brief, for adoptive transfer (passive EAE induction), donor mice were subcutaneously immunized with $\mathrm{MOG}_{35-55}$ peptide. Then draining lymph nodes and spleen were harvested 10 days after immunization. To prepare $\mathrm{MOG}_{35-55^{-}}$-specific 
polarized $\mathrm{T}$ cells, donor mice were immunized with MOG $_{35-55}$ subcutaneously, draining lymph node cells were prepared from donor mice 10 days after immunization. Cells were cultured for 5 days with $\mathrm{MOG}_{35-55}$ at a concentration of $10 \mu \mathrm{g} / \mathrm{ml}$ under Th1 $(20 \mathrm{ng} / \mathrm{ml} \mathrm{rmIL-12[R \& D],} 2$ $\mu \mathrm{g} / \mathrm{ml} \alpha \mathrm{IL}-23 \mathrm{p} 19$ [eBioscience]) polarizing conditions or Th17 (20 ng/ml rmIL-23[R\&D]) polarizing condition. Each recipient mouse was injected with $3.0 \times 10^{7}$ $M^{M O G}{ }_{35-55}$-specific Th1 or Th17 cells $4 \mathrm{~h}$ after 500 -Rad sub-lethal irradiation. Clinical scores were assessed in double-blind manner.

\section{Isolation of CNS inflammatory cells and flow cytometry}

Brains were homogenized in ice-cold tissue grinders, filtered through a $100-\mu \mathrm{m}$ cell strainer and the cells collected by centrifugation at $400 \times g$ for $5 \mathrm{~min}$ at $4{ }^{\circ} \mathrm{C}$. Cells were resuspended in $10 \mathrm{ml}$ of $30 \%$ Percoll (Amersham Bioscience) and centrifuge onto a 70\% Percoll cushion in $15-\mathrm{ml}$ tubes at $800 \times g$ for $30 \mathrm{~min}$. Cells at the $30-70 \%$ interface were collected and were subjected to flow cytometry. Fluorescence-conjugated CD4 (Clone GK1.5), CD8 (Clone 53-6.7), CD45 (Clone 30-F11), Ly6G (Clone 1A8) monoclonal antibodies and isotype controls were purchased from BD Biosciences. F4/80(Clone Cl: A3-1) was obtained from Serotech. The antibodies were diluted at 1:100 when used. Flow cytometry data were analyzed by FlowJo software.

\section{Histological analysis}

For paraffin-embedded tissue spinal cord removed from PBS perfused mice were fixed in $10 \%$ formalin. Sections were stained with either hematoxylin and eosin (H\&E) or myelin basic protein (MBP) to evaluate inflammation and demyelination, respectively. For frozen sections, spinal cords were embedded in OCT (Tissue-Tek) and snap frozen on liquid nitrogen. Sections $(10 \mu \mathrm{m}$ of thickness) were incubated with anti-CD4 antibody (BD Biosciences). Antigens were visualized following incubation with fluorescence-conjugated secondary antibodies (Molecular probe).

\section{ELISA}

IL-17 and IFN- $\gamma$ level were assayed by IL-17- or IFN- $\gamma$ ELISA kit (R\&D systems) following the manufacture's instruction.

\section{Real-time PCR}

Total RNA was extracted from spinal cord and cultured astrocytes with TRIzol (Invitrogen) according to the manufacture's instruction. All gene expression results are expressed as arbitrary units relative to expression of the gene encoding $\beta$-actin. Fold induction of gene expression in spinal cord after EAE induction was determined by dividing the relative abundance of experimental samples by the mean relative abundance of control samples from naïve mice.

\section{Statistics}

Non-parametric statistics was applied to all data set. The $p$ values of clinical scores were determined by two-way multiple-range analysis of variance (ANOVA) for multiple comparisons. Other $p$ values were determined by Mann-Whitney test unless otherwise specified. A $p$ value of $<0.05$ was considered significant. Unless otherwise specified, all results are shown as mean and the standard error of the mean (mean \pm SEM).

\section{Results}

STEAP4 promotes the pathogenesis of autoimmune encephalomyelitis

To identify the IL-17 target genes that function as effector molecules in the pathogenesis of EAE, we previously performed a transcriptomic profiling study on spinal cord tissues from wild-type and Act1-deficient (Act1 KO) mice that had received encephalitogenic Th17 cells [31]. Since Act1 deficiency abrogates IL-17 signaling, we reasoned that genes that were induced by encephalitogenic Th17 cells in wild-type but not Act1 knockout spinal cords were likely IL-17 target effector molecules. This led to the identification of STEAP4 as a top candidate IL-17 target effector during neuroinflammation (Fig. 1a). To determine the role of STEAP4 in the pathogenesis of EAE, we subjected STEAP4 knockout (STEAP4 KO) and wild-type littermate control mice to immunization with $M \mathrm{G}_{35-55}$ peptides to induce active EAE. Compared to wild-type littermate control, STEAP4 knockout mice exhibited markedly delayed disease onset (Fig. 1b). Moreover, while both STEAP4 knockout and littermate control mice reached $100 \%$ disease incidence 20 days after immunization (Supple. Fig. 1a), the clinical score and mean cumulative EAE scores of the STEAP4 knockout mice were lower compared to the controls (Fig. 1b and Supple. Fig. 2a). Consistent with the attenuated disease severity, histopathological analysis showed reduced infiltrating immune cell accumulation and resultant demyelination in spinal cord of the STEA P4 knockout mice compared to controls (Fig. 1c). Inflammatory mononuclear cell infiltration in the brain, including $\mathrm{T}$ cells, $\mathrm{B}$ cells, neutrophils, and macrophages, was consistently reduced in the STEAP4 knockout mice (Fig. 1d). Importantly, both the histological analysis and flow cytometric profiling were performed on the EAE mice 20 days post immunization, a time point when all mice had developed disease (Supple. Fig. 1a), indicating that the observed difference was not due to a difference in disease incidence but rather reflected an ameliorated disease severity in STEAP4 knockout mice. Collectively, these data suggested 


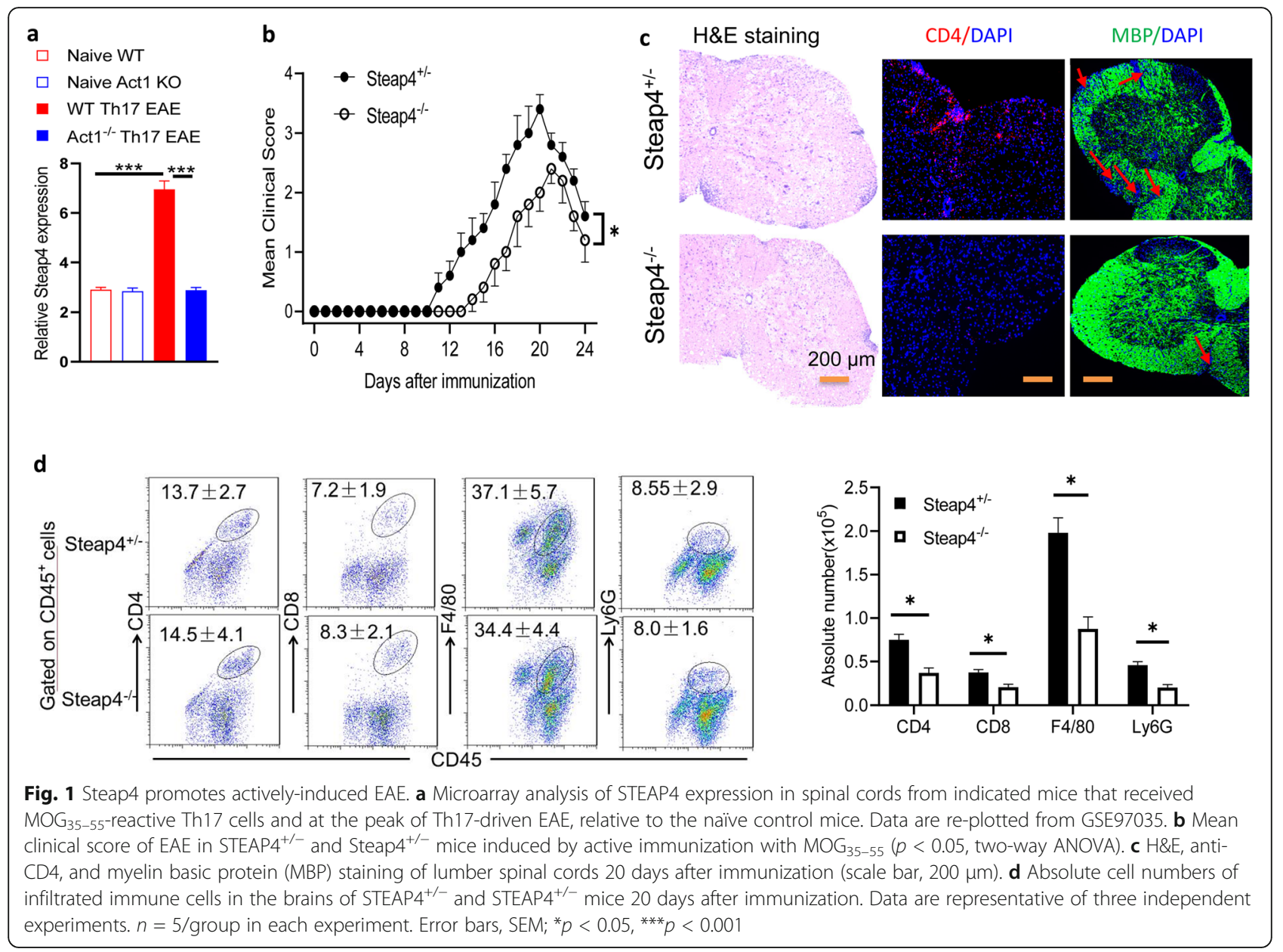

that STEAP4 promotes the pathogenesis of autoimmune encephalomyelitis and exacerbates neuroinflammation.

\section{STEAP4 deficiency in T cells does not impact their encephalitogenicity}

Current model suggests two distinct stages for the pathogenesis of active immunization-induced EAE. During the initiation stage, immunization induces encephalitogenic Th17 lineage lymphocytes, which subsequently infiltrates the central nervous system to mediate tissue damage in the effector stage of the disease [32, 33]. Given the phenotype of STEAP4 knockout mice in the active EAE model (Fig. 1b-d), we first asked whether STEAP4 plays a role in the differentiation of encephalitogenic Th17 cells. To answer this question, we immunized STEAP4 knockout and wild-type littermate control mice with MOG $_{35-55}$ peptides. Ten days after immunization, draining lymph node cells were harvested and culture under a non-polarizing condition (Th0) or condition that promotes the generation of Th1 or Th17 cells (Fig. 2a, b). Flow cytometric and ELISA analyses of the resultant cells indicated that STEAP4 deficiency did not impact the generation of Th1 or Th17 cells (Fig. 2a, b). Consistent with these findings, MOG-reactive Th17 cells from wild-type and STEAP4 knockout mice were equally encephalitogenic (Fig. 2c and Supple. Fig. 1b). Mice that received MOGreactive Th17 cells from STEAP4 knockout mice showed comparable levels of demyelination and immune cell infiltration to mice that received wild-type MOG-reactive $\mathrm{T}$ cells (Fig. 2d, e). Taken together, the data indicate that STEAP4 deficiency in $\mathrm{T}$ cells does not impact their encephalitogenicity.

\section{STEAP4 promotes Th17 cell-induced but not Th1 cell- induced autoimmune encephalomyelitis}

The expression of STEAP4 in the EAE spinal cord followed the pattern of clinical scores. STEAP4 mRNA was induced upon disease onset and it reached maximum at the peak of the disease (Supple. Fig. 3a). Intriguingly, the level of STEAP4 expression trended down among mice that started to recover (Supple. Fig. 3a). We sought to determine whether STEAP4 participates in the effector stage during the pathogenesis of EAE. Notably, while Th17 cells and its signature cytokine IL-17 is required for EAE induced by active immunization, MOG-reactive, differentiated Th1, and Th17 are both capable of driving 


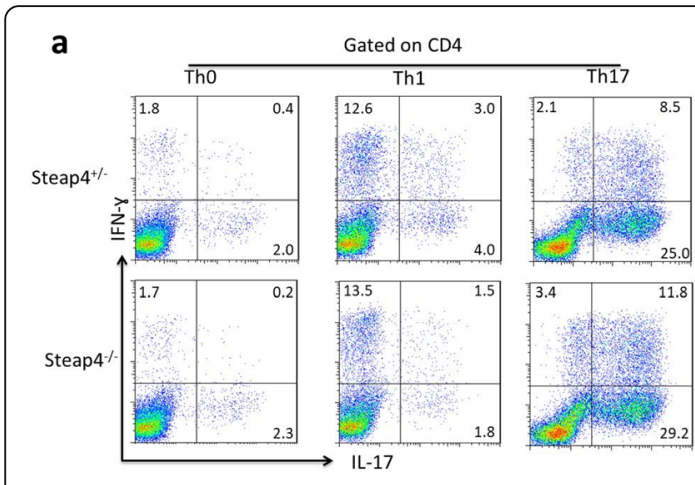

b

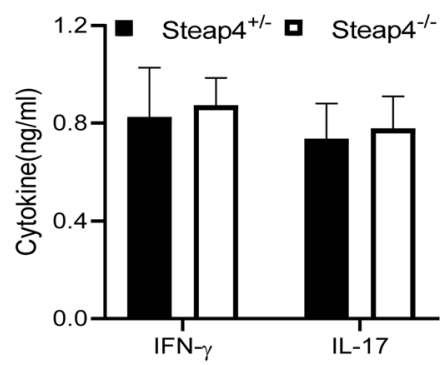

C

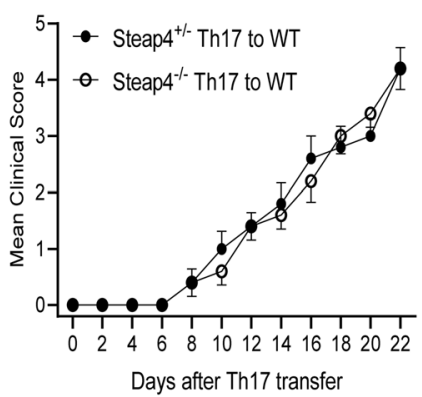

d

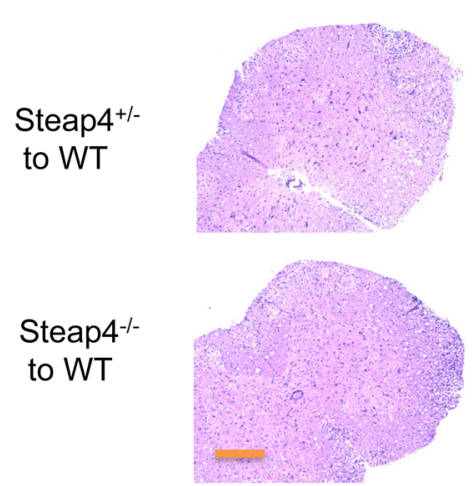

MBP/DAPI

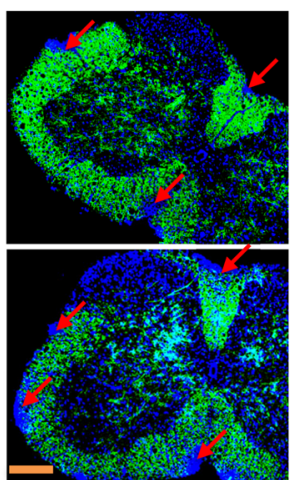

e

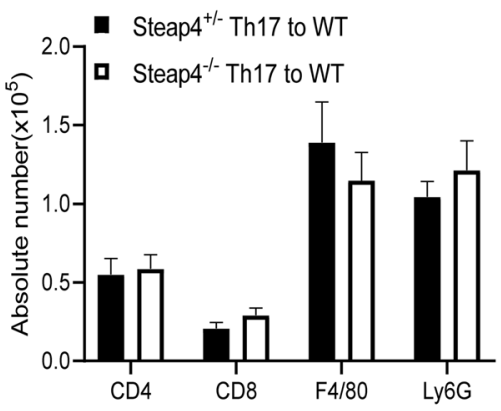

Fig. 2 STEAP4 ${ }^{-/-}$T cells are fully encephalitogenic. Mice were immunized by $\mathrm{MOG}_{35-55}$ and sacrificed 10 days after immunization. Lymph node cells were harvested and re-stimulated with MOG $35-55$ in DMEM medium alone (Th0), 20 ng/ml IL-12 (Th1), or 20 ng/ml IL-23 (Th17) for 5 days. a Data show the frequency of Th1 or Th17 cells under each polarization by intracellular staining. $\mathbf{b}$ Draining lymph node cells from wild-type mice and STEAP4deficient mice 10 days after immunization were re-stimulated with MOG $_{35-55}$ in vitro for 5 days, followed by ELISA of IL-17, IFN-ץ. c MOG $35-55^{-S p e c i f i c}$ Th17 cells from wild-type mice and STEAP4-deficient mice were used as donor cells and transferred to naïve wild-type recipient mice. Graph represents the mean clinical score after Th17-cell transfer. $\mathbf{d}$ H\&E and myelin basic protein (MBP) staining of lumber spinal cords at the end point of EAE. e Absolute cell numbers of infiltrated immune cells in the brains of recipients of STEAP4 ${ }^{+/}$Th17 or recipients of STEAP4 ${ }^{-/}$Th17 cells. Data are representative of three independent experiments. $n=5 /$ group in each experiment. Error bars, SEM; ${ }^{*} p<0.05,{ }^{* *} p<0.01$

neuroinflammation when they are adoptively transferred into sublethally irradiated recipient mice. Importantly, encephalitogenic Th17 but not Th1 cells induced the expression of STEAP4 in the spinal cord of recipient mice [34, 35] (Fig. 3a, Supple. Fig. 1d), suggesting that STEAP4 is a Th17-specific effector molecule in the pathogenesis of EAE. To test this idea, we transferred either MOG-reactive Th17 or MOG-reactive Th1 cells into wild-type and STEAP4 knockout mice (Fig. 3b-h). Consistent with the specific induction of STEAP4 by Th17 cells, the disease severity of STEAP4 knockout mice was substantially reduced compared to that of the wild-type littermate control (Fig. 3b). The amelioration of EAE symptoms in the STEAP4 knockout mice was characterized by delayed onset and peak of disease as well as a dampened cumulative clinical score (Fig. 3b, Supple. Figs. 1 c, 2 b). In line with the clinical score, STEAP4 knockout mice also sustained less demyelination in their spinal cords (Fig. 3c) and had less immune cell infiltration in the brain compared to littermate controls
(Fig. 3d). The expression of inflammatory cytokine and chemokine expression in the spinal cord was also significantly decreased (Fig. 3e). In contrast, MOG-reactive Th1 cells induced comparable disease in both STEAP4 knockout and wild-type littermate controls (Fig. 3f-h, Supple. Fig. 1d). Taken together, the data suggest that STEAP4 is an effector molecule in Th17- but not Th1-driven EAE.

STEAP4 expression in neuroectoderm-derived CNS resident cells is required for the pathogenesis of active EAE

We have previously shown that IL-17-induced Act1mediated signaling in neuroectoderm-derived cells in the central nervous system (CNS) is critical for the pathogenesis of EAE $[16,17]$. In addition, we reported that IL-17 readily induced STEAP4 expression in a number of tissue cells. These evidences led us to hypothesize that STEAP4 expression in the CNS resident cells is required for active EAE pathogenesis. To test this hypothesis, we generated 


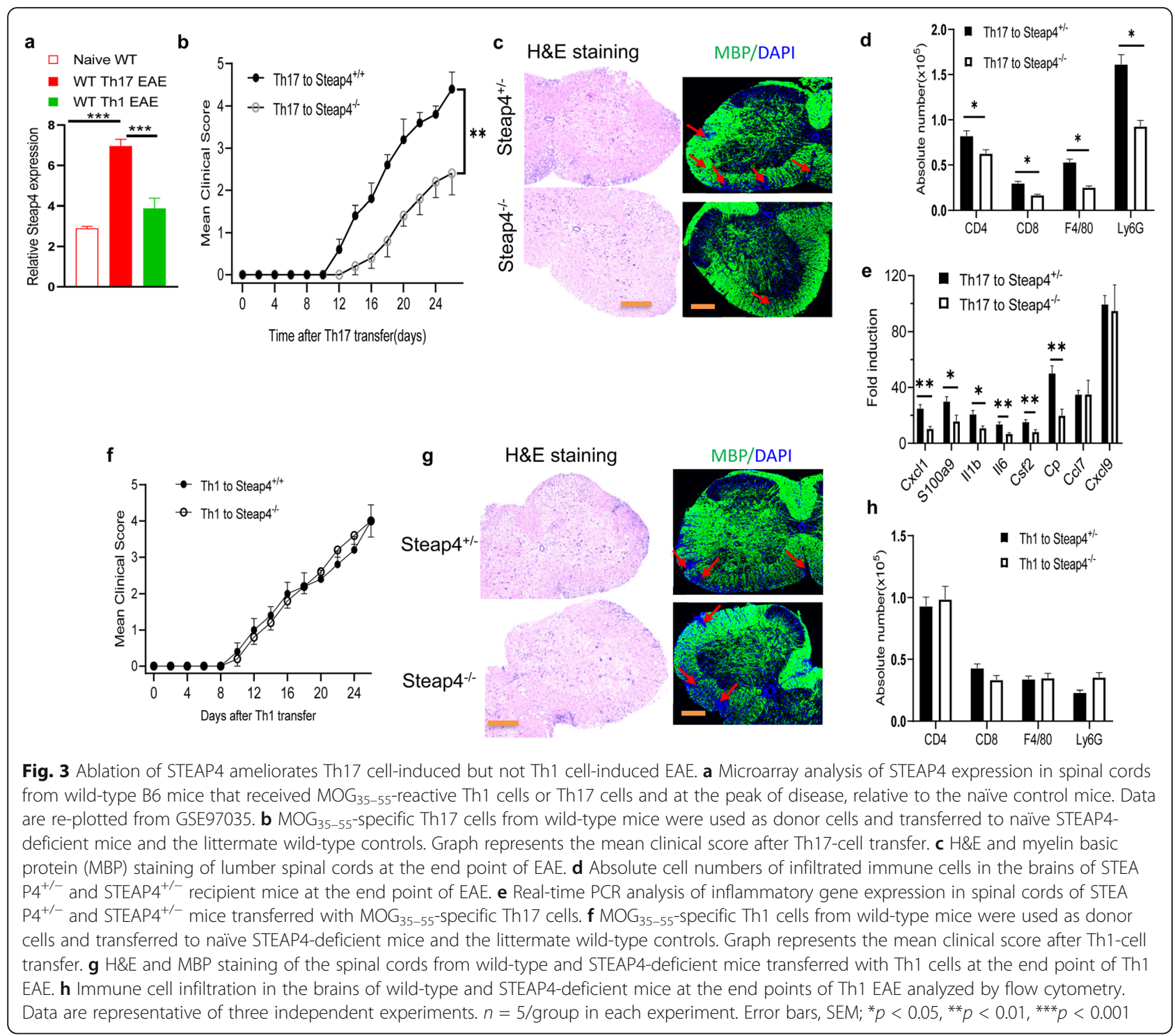

Nestin-Cre, STEAP4 ${ }^{\mathrm{f} / \mathrm{fl}}$ mice. Nestin-Cre is expressed in all neuroectoderm-derived cells of the central nervous system, resulting in ablation of LoxP-flanked sequences in all CNS resident cells including astrocytes, neuron and oligodendrocyte lineage cells. Specific STEAP4 ablation was confirmed by western blot in astrocytes from Nestin-Cre STEAP4 $4^{\mathrm{Al}++}$ and Nestin-Cre STEAP4 ${ }^{\mathrm{I} / \mathrm{ll}}$ neonatal pups (Fig. 4a). We induced EAE in Nestin-Cre STEAP4 ${ }^{\text {I/+ }}$ and Nestin-Cre STEA $P 4^{f / f l}$ mice by active immunization with $\mathrm{MOG}_{35-55}$ peptides. Similar to the phenotype observed in the STEAP4 global knockout mice, Nestin-Cre STEAP4 ${ }^{f l / l}$ mice showed a delayed onset of disease and reduced cumulative clinical scores compared to Nestin-Cre STEAP4 ${ }^{t /+}$ control mice, but mice in both groups had $100 \%$ disease incidence (Fig. 4b, c, Supple. Fig. 2c). The reduction in disease severity was associated with a markedly reduced demyelination and immune cell infiltration (Fig. 4d, e). To determine the contribution of STEAP4 expression from CNS-resident cells during EAE, we analyzed the level of STEAP4 expression in spinal cords from Nestin-Cre STEAP4 fl/fl and littermate control EAE mice early at disease onset when mice with comparable disease severity (clinical score $=1 \sim 2$ ) were available from both groups. The data showed that Nestin-Cre mediated deletion reduced the expression of STEAP4 by about $70 \%$ in the spinal cord (Supple. Fig. 3B). Furthermore, the expression of STEAP4 in sorted neutrophil (CD11b Ly6G+) and monocytes (CD11b Ly6C+ Ly6G-) from Nestin-Cre STEAPf $f^{f l / f l}$ and littermate control EAE mice were comparable (Supple. Fig. 3C), indicating that the Creexpression did not leak into the myeloid-lineage. Together these data suggest that STEAP4 expression in 


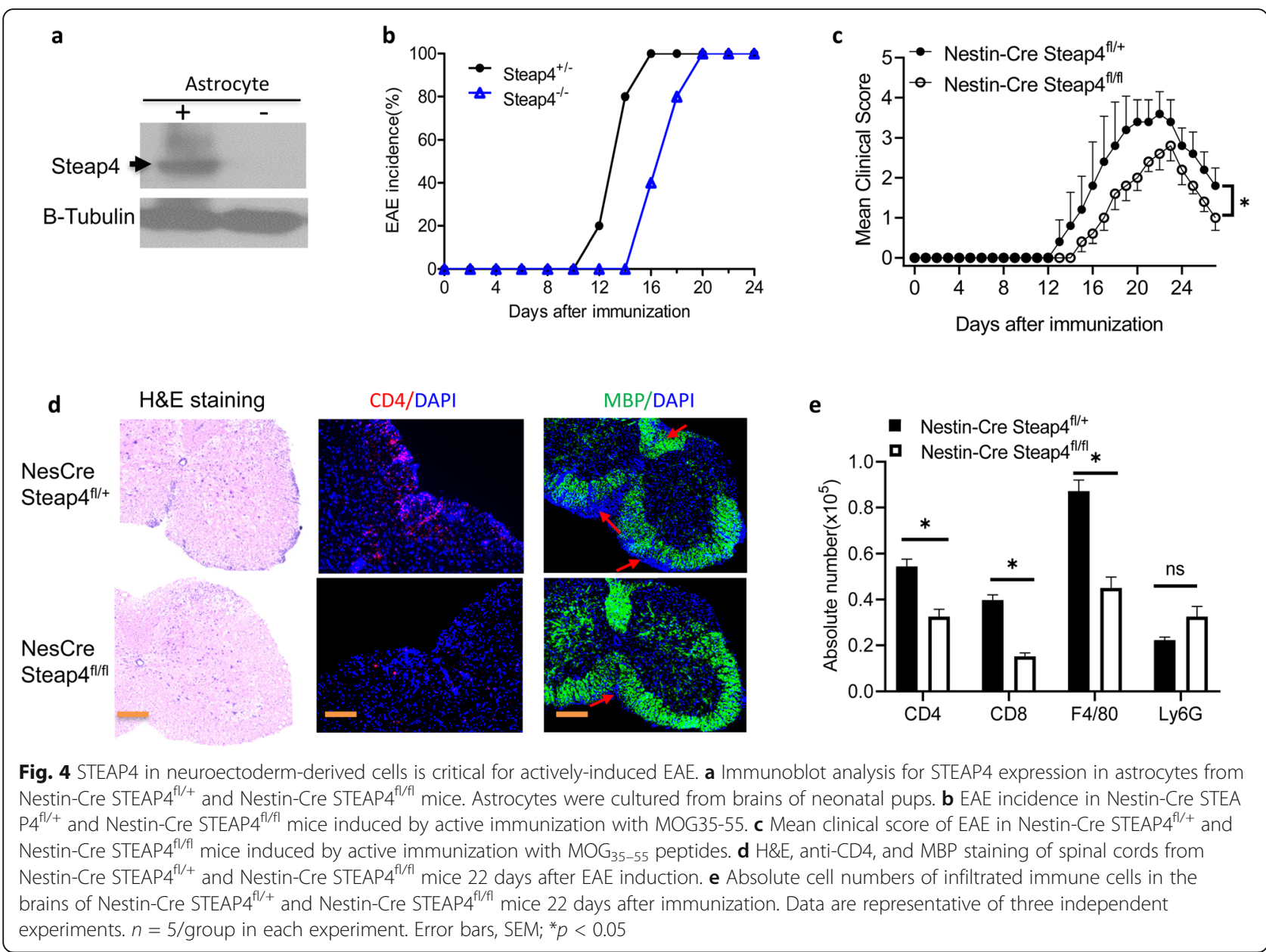

CNS resident cells is required for the pathogenesis of active EAE.

\section{STEAP4 in CNS resident cells promote Th17- but not Th1- induced EAE}

Since our data suggested that STEAP4 is a specific effector molecule for Th17- but not Th1-induced EAE (Fig. 3), we examined whether the pathogenic impact was predominantly contributed by STEAP4 expression in CNS resident $\mathrm{T}$ cells. Using a similar experimental design, we transferred MOG-reactive Th17 or Th1 cells into Nestin-Cre STEAP4 $4^{\mathrm{fl} / \mathrm{fl}}$ mice and littermates NestinCre STEAP $4^{\mathrm{fl} /+}$ mice. Consistent with the phenotype observed in the global STEAP4 knockout mice, the disease onset was much delayed and plateau clinical score was significantly reduced in Nestin-Cre STEAP4 ${ }^{f l f l}$ mice that received MOG-reactive Th17 cells compared to NestinCre STEAP4 $4^{f l+}$ control recipients though both groups has $100 \%$ EAE incidence, consistently the cumulative EAE score was also dramatically reduced in Nestin-Cre STEA $P 4^{f l / f l}$ mice (Fig. 5a, Supple. Figs. 1e, 2d). Demyelination, immune cell infiltration and expression of inflammatory genes were consistently reduced in the Nestin-Cre STEA
$P 4^{f l / f l}$ recipients (Fig. 5b-d). In contrast, the onset of disease was simultaneous and the peak clinical score was comparable in Nestin-Cre STEAP4 $4^{\mathrm{fl} / \mathrm{fl}}$ and littermate Nestin-Cre STEAP4 ${ }^{f l+}$ mice that received MOG-reactive Th1 cells, resulting in similar levels of demyelination and immune cell infiltration (Fig. 5e-g, Supple. Fig. 1f). Collectively, the data indicate that STEAP4 is an effector molecule in CNS resident cells during Th17- but not Th1-induced EAE.

\section{Discussion}

In this study, we identified STEAP4 as a Th17-specific effector molecule that participates and contributes to the pathogenesis of neuroinflammation. While MOGreactive Th17 cells induced STEAP4 expression in the spinal cord of EAE mice, STEAP4 deficiency attenuated the onset of EAE induced by active immunization. Our data indicated that the reduced pathogenesis in STEAP4 knockout mice was unlikely due to the impact of STEA P4 on T cells, as the induction of MOG-reactive Th17 cells in STEAP4 knockout mice was intact and those cells exhibited normal encephalitogenicity when transferred into recipient mice, pointing to a role for STEAP4 


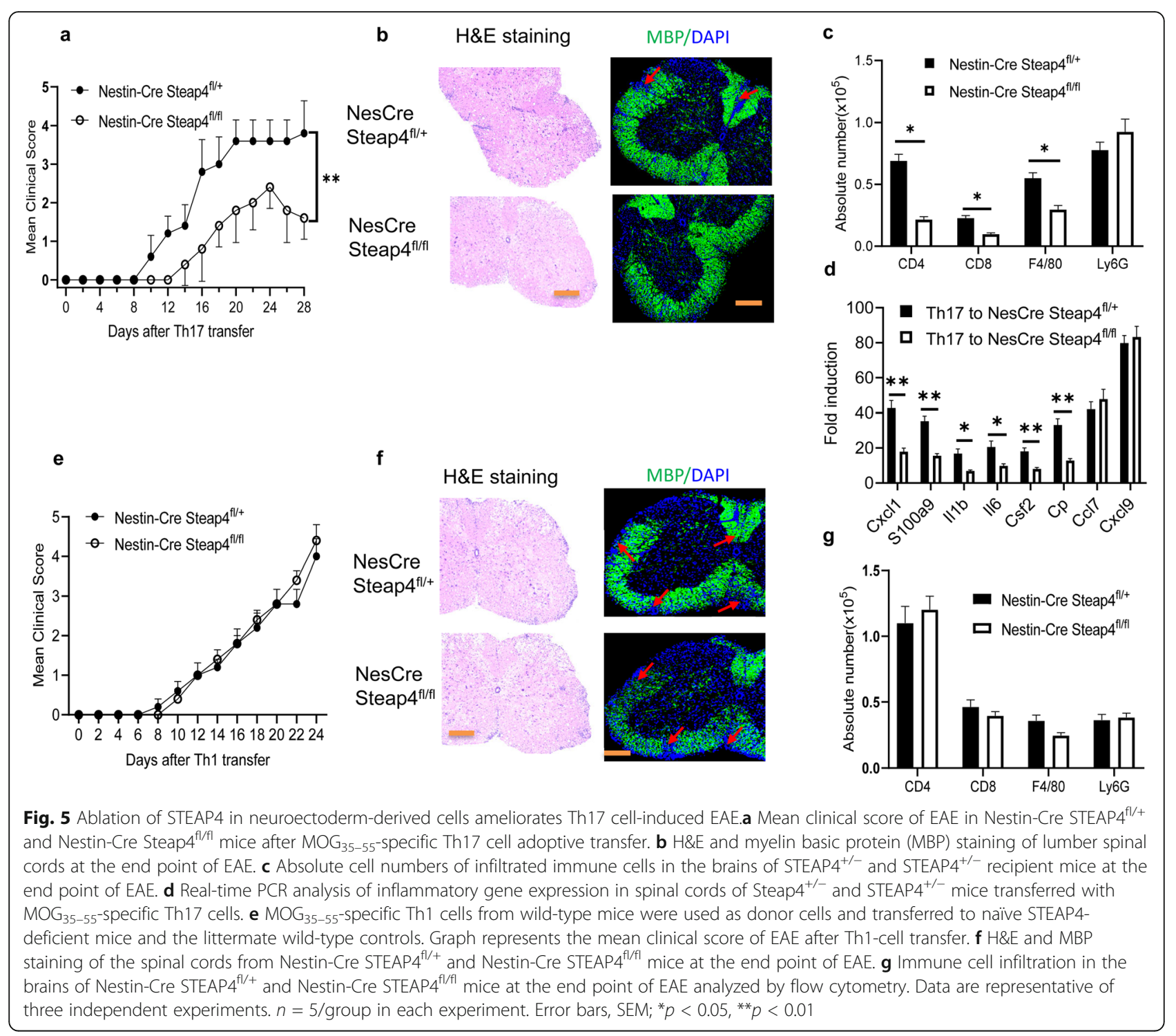

in the effector stage of the pathogenesis. Indeed, STEA $P 4$ knockout mice were relatively resistant to passively induced EAE by MOG-reactive Th17 cells. Importantly, while STEAP4 was specifically induced by MOG-reactive Th17 cells but not Th1 cells, STEAP4 deficiency only ameliorated the EAE induced by MOG-reactive Th17 cells but not Th1 cells. This observation is consistent with our previous report showing that STEAP4 is an IL-17 target gene. This notion was further substantiated by our finding that STEAP4 expression in the CNS resident cells was critical for both active immunization-induced and MOG-reactive Th17 cells-mediated neuroinflammation.

The identification of a Th17-induced and Th17specific effector molecule for EAE pathogenesis has major implications. The finding suggest that while both myelin-reactive Th1 and Th17 cells can mediate tissue destruction and demyelination, the underlying molecular process may be drastically different. Indeed, previous study has shown that the disease kinetics and pathologies of EAE elicited by different $\mathrm{T}$ helper cell subset or lineages, including Th1, Th17, and Th9 cells [36], have distinct characteristics. Appreciation of the diverging underlying pathogenic mechanism may be critical for our understanding of the broad and extensive heterogeneity in terms of clinical features, genetics, pathogenesis, and responsiveness to treatments in MS patients. In this regard, immunohistochemical analysis of STEAP4 and IL-17 expression in post-mortem brain specimen from MS patients is warranted. In addition, a large number of MS cases are characterized by a relapse-remitting disease course. A key challenge in management of MS is disease relapse. As our data suggest that STEAP4 expression appeared to trend down in mice that started to recover, an important question is whether STEAP4 plays a role 
in the remission and relapse of neuroinflammation. Future studies using PLP-immunization in SJL mice are required to fully address this question.

A key question raised by our in vivo analysis is how STEAP4 participates and contributes to Th17-mediated demyelination. STEAP4 is an inflammatory metalloreductase that catalyzes the reduction of copper and iron and the oxidation of NADPH. We and others have shown that the expression of STEAP4 promotes the cellular uptake of copper and iron, as only the reduced form of both metal ion are transported across the cellular membrane [24, 25, 37]. We recently reported that increased level of intracellular copper enhances and sustains the activation of $\mathrm{NFKB}$, resulting in elevated production of inflammatory cytokine and chemokines [25]. This STEAP4-mediated induction of chemokine and cytokines would contribute to increased recruitment and activation of immune cells, which eventually lead to tissue damage in the CNS.

On the other hand, the activity of STEAP4 also depletes NADPH and produces NADP+ [24]. NADPH, a potent antioxidant, plays a fundamental role in regulating the intracellular redox balance. NADPH is the ultimate electron donor for most of the detoxifying enzymes that remove harmful reactive oxygen species (ROS), the culprit metabolites for oxidative stress. While moderate level of ROS has been associated with proliferative signals and potentiate inflammatory cytokine production, excessive oxidative stress can lead to cell death. The potential role of STEAP4-mediated ROS production has two implications based on our findings. Microglia, like other immune cells such as macrophages, are likely more equipped to handle oxidative stress. Hence, increased ROS production in microglia may help to amplify the inflammatory response by promoting the production of cytokines and chemokines. However, oligodendrocytes were said to be particularly vulnerable to oxidative stress [38]. Oxidative stress has also been suggested to inhibit oligodendrocyte differentiation [39]. Thus, it is plausible that STEAP4 expression in oligodendrocyte lineage cells may promote direct demyelination and inhibits re-myelination.

In the current study, we primarily focused on the CNS-resident cells because our previous study showed that the CNS-resident cells, and specifically oligodendrocyte progenitor cells, were the target cells for IL-17. While STEAP4 was indeed induced by IL-17 in the CNS-resident cells, myeloid cells, in particular neutrophils, were known to highly express STEAP4 $[25,40]$. Notably, the infiltration of macrophages and neutrophils were reduced in the STEAP4 knockout mice in the active EAE model. However, such reduction was less pronounced in the Nestin-Cre STEA $\mathrm{P} 4^{\mathrm{fl} / \mathrm{fl}}$ mice, suggesting additional role for STEAP4 in myeloid cells. Nevertheless, whether and how STEAP4 expression in myeloid cells contributes to the pathogenesis of EAE requires further study using cell type specific knockouts.

\section{Conclusion}

In this study, we identified STEAP4 as a Th17-specific effector molecule that participates and contributes to the pathogenesis of neuroinflammation. STEAP4 knockout mice displayed delayed onset and reduced severity of EAE induced by active immunization. The reduced disease phenotype was not due to any impact of STEAP4 deficiency on myelin reactive $\mathrm{T}$ cells. Importantly, STEA $P 4$ was specifically induced by MOG-reactive Th17 cells but not Th1 cells, STEAP4 deficiency only ameliorated the EAE induced by MOG-reactive Th17 cells but not Th1 cells. By using Nestin-Cre STEAP4 ${ }^{\mathrm{fl} / \mathrm{fl}}$ mice, we showed that ablation of STEAP4 expression in the resident cells in the central nervous system attenuated disease severity in both active immunization and passive Th17 transfer-induced EAE, thus suggesting a CNSintrinsic role of STEAP4 in the pathogenesis of autoimmune neuroinflammation. Since STEAP4 is also highly expressed in myeloid cells, whether and how STEAP4 expression in myeloid cells contributes to the pathogenesis of EAE requires further study.

\section{Abbreviations}

CNS: Central nervous system; EAE: Experimental autoimmune encephalomyelitis; MS: Multiple sclerosis; Th: T helper cell; H\&E: Hematoxylin and eosin; MBP: Myelin basic protein; MOG: Myelin oligodendrocyte glycoprotein; Act1: Nuclear factor NFkappa-B activator 1; STEAP4: Six transmembrane epithelial antigen of prostate; ELISA: Enzyme-linked immunosorbent assay

\section{Supplementary Information}

The online version contains supplementary material available at https://doi. org/10.1186/s12974-021-02146-7.

\begin{abstract}
Additional file 1: Supplementary Figure S1. EAE incidence after active or passive EAE induction. (a) Steap $4^{+/-}$and Steap $4^{-/-}$mice were induced to develop EAE by active immunization with $\mathrm{MOG}_{35-55}$; Disease incidence are shown. Related to Fig.1 b; related to Fig. 1 b; (b). MOG 35 -55-specific Th17 cells from Steap $4^{+/}$and Steap ${ }^{-/}$mice were used as donor cells and transferred to naïve wild-type recipient mice. Graph represents EAE incidence after $\mathrm{MOG}_{35-55}$-specific Th17-cell transfer, related to Fig. 2 c; (c)

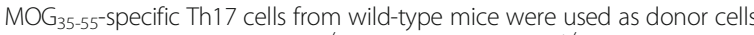
and transferred to naïve Steap $4^{-/-}$mice and the Steap $4^{+/-}$littermate controls. Graph represents the EAE incidence after $\mathrm{MOG}_{35-55}$-specific Th17-cell transfer, related to Fig. 3 b; (d) MOG $35-55$-specific Th1 cells from wild-type mice were used as donor cells and transferred to naïve Steap4 ${ }^{+/}$and Steap4 ${ }^{-/}$ mice. Graph represents the EAE incidence after Th1-cell transfer, related to Fig. $3 \mathrm{f}$; (e) EAE incidence in Nestin-Cre Steap4 ${ }^{\mathrm{f} / /+}$ and Nestin-Cre Steap4 ${ }^{\mathrm{f} / / \mathrm{fl}}$ mice are shown after $\mathrm{MOG}_{35-55}$-specific Th17 cell adoptive transfer, related to Fig. 5 a; (f) EAE incidence in Nestin-Cre Steap4 ${ }^{f /+}$ and Nestin-Cre Steap4 $4^{f / f l}$ mice are shown after $\mathrm{MOG}_{35-55}$-specific Th1 cell adoptive transfer, related to Fig. 5 e. Data are representative of three independent experiments. $n=5$ / group in each experiment. $p$ values were determined by Log-rank test and shown in each panel.
\end{abstract}

Additional file 2: Supplementary Figure S2. Mean cumulative scores of EAE after active or passive induction. (a) Steap4 $4^{+/}$and Steap4 $4^{-/-}$mice were induced to develop EAE by active immunization with $\mathrm{MOG}_{35-55}$; 
Mean cumulative scores are shown. Related to Fig. 1 b; (b) Steap4 $4^{+/}$and Steap $4^{-1-}$ mice were induced to develop EAE by $\mathrm{MOG}_{35-55}$-specific Th17 cell adoptive transfer, Mean cumulative scores are shown. Related to Fig. 3 b; (c) Nestin-Cre Steap $4^{f / /+}$ and Nestin-Cre Steap $4^{f / / f}$ mice were induced to develop EAE by active immunization with $\mathrm{MOG}_{35-5}$; Mean cumulative scores are shown. Related to Fig. 4 c; (d) Nestin-Cre Steap4 ${ }^{\mathrm{fl} /+}$ and NestinCre Steap $4^{\mathrm{f} / \mathrm{fl}}$ mice were induced to develop EAE by $\mathrm{MOG}_{35-55}$-specific Th17 cell adoptive transfer, Mean cumulative scores are shown. Related to Fig. 5 a. Data are representative of three independent experiments. $n=$ 5/group in each experiment. Error bars, SEM. p values were determined by Mann-Whitney test and shown in each panel.

Additional file 3: Supplementary Figure S3. Expression pattern and deletion specificity of Steap4. (a) Spinal cords were harvested from MOG immunized mice at pre-symptomatic stage (clinical score $=0$ ), disease onset (clinical score 2 3), peak of the disease (clinical score 4 5) and remission (clinical score 4 3). Harvested spinal cords were analyzed for Steap4 expression by RT-PCR. The average $2^{-\Delta C t}$ values of Steap4 in pre-

symptomatic spinal cords were set as 1 . The fold changes in spinal cords from disease onset, peak of disease and remission were calculated by divide the $2^{-\Delta \mathrm{Ct}}$ value of individual biological sample (a spinal cord) by the average $2^{-\Delta C t}$ values of Steap4 in pre-symptomatic spinal cords, which is set as 1. The $P$ value for one-way ANOVA analysis is $<0.0001$ (smaller than the software limit). Statistically significant $P$ values for post hoc two-sided unpaired $t$ test between groups are indicated on the figure. Error bars, SEM (b) Spinal cords from EAE mice of indicated genotype with a clinical score of 1 2 were harvested and analyzed for Steap 4 expression by RT$P C R$. The average $2^{-\Delta C t}$ values of Steap 4 in Nestin-Cre Stea $4 \mathrm{fl} /+$ spinal cords were set as 1 . Fold change were calculated according the formula described for panel (a). Two-sided unpaired t test was employed to compute the $P$ value, which was smaller than 0.0001 beyond software limit. Error bars, SEM. (c-d) Splenocytes from mice of indicated genotype were harvested and sorted by FACS to obtain CD11b+Ly6G+ cells and CD11b+Ly6C+Ly6G- cells. Sorted cells were analyzed for Steap4 expression by RT-PCR, $n=3 \sim 5 /$ group in each experiment. The average $2^{-\Delta C}$ values of Steap4 in Nestin-Cre Stea $4 \mathrm{fl} /+$ spinal cords were set as 1. Fold change were calculated according the formula described for panel. Error bars, SEM. $P$ value by one way ANOVA in (c) is 0.0018 . $P$ value by one way ANOVA in (d) is 0.0010 . Statistically significant $P$ values for post hoc unpaired two-sided t test between groups are indicated on the figure.

\section{Acknowledgements}

Not applicable

\section{Authors' contributions}

$J J Z$ designed and performed most of the experiments, interpreted the data $Y L$ did the mouse breeding; MLW did the genotyping and gene expression assay; JX did the western blot and PCR; CL performed some immunohistochemical staining. Xiaoxia Li contributed to experimental design and manuscript writing. Xiao Li did the microarray analysis, experiment design, data interpretation, and manuscript writing; ZK was integral for experimental design, manuscript writing, data interpretation, and project coordination. All authors read and approved the final manuscript.

\section{Funding}

This investigation was supported by grants from the US National Institutes of Health (R01NS104164 to Z.K.), by Case Western Reserve University through departmental startup funding awarded to Xiao Li and NMSS (RG-1707-28180 to Xiaoxia Li).

\section{Availability of data and materials}

All datasets and analyses used in the current study are available from the corresponding author on reasonable request.

\section{Declarations}

\section{Ethics approval and consent to participate}

All procedures were approved by the Institutional Animal Care and Use Committee of Cleveland Clinic and conducted in accordance with the animal care guidelines of the National Institute of Health.

\section{Consent for publication}

Not applicable

\section{Competing interests}

The authors declare that they have no competing interests.

\section{Author details}

${ }^{1}$ Department of Inflammation and Immunity, Lerner Research Institute, Cleveland, OH, USA. ${ }^{2}$ The Center for RNA Science and Therapeutics, Case Western Reserve University, Cleveland, OH, USA. ${ }^{3}$ Department of Pathology, University of lowa, lowa City, IA 52242, USA.

Received: 4 December 2020 Accepted: 1 April 2021

Published online: 20 April 2021

\section{References}

1. Ransohoff RM. Animal models of multiple sclerosis: the good, the bad and the bottom line. Nat Neurosci. 2012;15(8):1074-7. https://doi.org/1 $0.1038 / \mathrm{nn} .3168$.

2. Stromnes IM, Goverman JM. Passive induction of experimental allergic encephalomyelitis. Nat Protoc. 2006;1(4):1952-60. https://doi.org/10.1038/ nprot.2006.284.

3. Stromnes IM, Goverman JM. Active induction of experimental allergic encephalomyelitis. Nat Protoc. 2006;1(4):1810-9. https://doi.org/10.1038/ nprot.2006.285.

4. Thompson AJ, Baranzini SE, Geurts J, Hemmer B, Ciccarelli O. Multiple sclerosis. Lancet. 2018;391(10130):1622-36. https://doi.org/10.1016/S01406736(18)30481-1.

5. Sospedra M, Martin R. Immunology of multiple sclerosis. Semin Neurol. 2016;36(02):115-27. https://doi.org/10.1055/s-0036-1579739.

6. Becher B, Bechmann I, Greter M. Antigen presentation in autoimmunity and CNS inflammation: how T lymphocytes recognize the brain. J Mol Med (Berl). 2006;84(7):532-43. https://doi.org/10.1007/s00109-006-0065-1.

7. Mangan PR, Harrington LE, O'Quinn DB, Helms WS, Bullard DC, Elson CO, et al. Transforming growth factor-beta induces development of the $\mathrm{T}(\mathrm{H}) 17$ lineage. Nature. 2006:441(7090):231-4. https://doi.org/10.1038/nature04754.

8. Veldhoen M, Hocking RJ, Atkins CJ, Locksley RM, Stockinger B. TGFbeta in the context of an inflammatory cytokine milieu supports de novo differentiation of IL-17-producing T cells. Immunity. 2006;24(2):179-89. https://doi.org/10.1016/j.immuni.2006.01.001.

9. Cua DJ, Sherlock J, Chen Y, Murphy CA, Joyce B, Seymour B, et al. Interleukin-23 rather than interleukin-12 is the critical cytokine for autoimmune inflammation of the brain. Nature. 2003;421(6924):744-8. https://doi.org/10.1038/nature01355.

10. Ivanov II, McKenzie BS, Zhou L, Tadokoro CE, Lepelley A, Lafaille JJ, et al. The orphan nuclear receptor RORgammat directs the differentiation program of proinflammatory IL-17+ T helper cells. Cell. 2006;126(6):1121-33. https://doi. org/10.1016/j.cell.2006.07.035

11. Kang Z, Liu L, Spangler R, Spear C, Wang C, Gulen MF, et al. IL-17induced Act1-mediated signaling is critical for cuprizone-induced demyelination. J Neurosci. 2012;32(24):8284-92. https://doi.org/10.1523/ JNEUROSCI.0841-12.2012.

12. Hu Y, Ota N, Peng I, Refino CJ, Danilenko DM, Caplazi P, et al. IL-17RC is required for IL-17A- and IL-17F-dependent signaling and the pathogenesis of experimental autoimmune encephalomyelitis. J Immunol. 2010;184(8): 4307-16. https://doi.org/10.4049/jimmunol.0903614.

13. Komiyama Y, Nakae S, Matsuki T, Nambu A, Ishigame H, Kakuta S, et al. IL-17 plays an important role in the development of experimental autoimmune encephalomyelitis. J Immunol. 2006;177(1):566-73. https://doi.org/10.4049/ jimmunol.177.1.566.

14. Qian Y, Liu C, Hartupee J, Altuntas CZ, Gulen MF, Jane-Wit D, et al. The adaptor Act1 is required for interleukin 17-dependent signaling associated with autoimmune and inflammatory disease. Nat Immunol. 2007:8(3):247_ 56. https://doi.org/10.1038/ni1439.

15. Li X, Commane M, Nie H, Hua X, Chatterjee-Kishore M, Wald D, et al. Act1, an NF-kappa B-activating protein. Proc Natl Acad Sci U S A. 2000;97(19): 10489-93. https://doi.org/10.1073/pnas.160265197.

16. Kang Z, Wang C, Zepp J, Wu L, Sun K, Zhao J, et al. Act1 mediates IL-17induced EAE pathogenesis selectively in NG2+ glial cells. Nat Neurosci. 2013;16(10):1401-8. https://doi.org/10.1038/nn.3505. 
17. Kang Z, Altuntas CZ, Gulen MF, Liu C, Giltiay N, Qin H, et al. Astrocyterestricted ablation of interleukin-17-induced Act1-mediated signaling ameliorates autoimmune encephalomyelitis. Immunity. 2010;32(3):414-25. https://doi.org/10.1016/j.immuni.2010.03.004.

18. Havrdova E, Belova A, Goloborodko A, Tisserant A, Wright A, Wallstroem E, et al. Activity of secukinumab, an anti-IL-17A antibody, on brain lesions in RRMS: results from a randomized, proof-of-concept study. J Neurol. 2016; 263(7):1287-95. https://doi.org/10.1007/s00415-016-8128-x.

19. Hubert RS, Vivanco I, Chen E, Rastegar S, Leong K, Mitchell SC, et al. STEAP: a prostate-specific cell-surface antigen highly expressed in human prostate tumors. Proc Natl Acad Sci U S A. 1999;96(25):14523-8. https://doi.org/10.1 073/pnas.96.25.14523.

20. Oosterheert W, van Bezouwen LS, Rodenburg RNP, Granneman J, Forster F, Mattevi A, et al. Cryo-EM structures of human STEAP4 reveal mechanism of iron(III) reduction. Nat Commun. 2018;9(1):4337. https://doi.org/10.1038/s414 67-018-06817-7.

21. Kim K, Mitra S, Wu G, Berka V, Song J, Yu Y, et al. Six-transmembrane epithelial antigen of prostate 1 (STEAP1) Has a single $b$ heme and is capable of reducing metal ion complexes and oxygen. Biochemistry. 2016 55(48):6673-84. https://doi.org/10.1021/acs.biochem.6b00610.

22. Ohgami RS, Campagna DR, McDonald A, Fleming MD. The Steap proteins are metalloreductases. Blood. 2006;108(4):1388-94. https://doi.org/10.1182/ blood-2006-02-003681.

23. Ohgami RS, Campagna DR, Greer EL, Antiochos B, McDonald A, Chen J, et al. Identification of a ferrireductase required for efficient transferrindependent iron uptake in erythroid cells. Nat Genet. 2005;37(11):1264-9. https://doi.org/10.1038/ng1658

24. Xue X, Bredell BX, Anderson ER, Martin A, Mays C, Nagao-Kitamoto H, et al. Quantitative proteomics identifies STEAP4 as a critical regulator of mitochondrial dysfunction linking inflammation and colon cancer. Proc Natl Acad Sci U S A. 2017;114(45):E9608-17. https:/doi.org/10.1073/pnas.1712946114.

25. Liao Y, Zhao J, Bulek K, Tang F, Chen X, Cai G, et al. Inflammation mobilizes copper metabolism to promote colon tumorigenesis via an IL-17-STEAP4XIAP axis. Nat Commun. 2020;11(1):900. https://doi.org/10.1038/s41467-02 0-14698-y.

26. Cherner JA, Cloud ML, Offen WW, Latz JE. Comparison of nizatidine and cimetidine as once-nightly treatment of acute duodenal ulcer. Nizatidine Multicenter Duodenal Ulcer Study Group. Am J Gastroenterol. 1989;84:769-74

27. Wellen KE, Fucho R, Gregor MF, Furuhashi M, Morgan C, Lindstad T, et al. Coordinated regulation of nutrient and inflammatory responses by STAMP2 is essential for metabolic homeostasis. Cell. 2007;129(3):537-48. https://doi. org/10.1016/j.cell.2007.02.049.

28. ten Freyhaus $\mathrm{H}$, Calay ES, Yalcin A, Vallerie SN, Yang L, Calay ZZ, et al Stamp2 controls macrophage inflammation through nicotinamide adenine dinucleotide phosphate homeostasis and protects against atherosclerosis. Cell Metab. 2012;16(1):81-9. https://doi.org/10.1016/j. cmet.2012.05.009.

29. Giusti SA, Vercelli CA, Vogl AM, Kolarz AW, Pino NS, Deussing JM, et al. Behavioral phenotyping of Nestin-Cre mice: implications for genetic mouse models of psychiatric disorders. J Psychiatr Res. 2014;55:87-95. https://doi. org/10.1016/j.jpsychires.2014.04.002.

30. Tronche F, Kellendonk C, Kretz O, Gass P, Anlag K, Orban PC, et al. Disruption of the glucocorticoid receptor gene in the nervous system results in reduced anxiety. Nat Genet. 1999;23(1):99-103. https://doi.org/10.1 038/12703.

31. Wang C, Zhang CJ, Martin BN, Bulek K, Kang Z, Zhao J, et al. IL-17 induced NOTCH1 activation in oligodendrocyte progenitor cells enhances proliferation and inflammatory gene expression. Nat Commun. 2017;8(1): 15508. https://doi.org/10.1038/ncomms15508.

32. Ransohoff RM. Immunology: In the beginning. Nature. 2009;462(7269):41-2. https://doi.org/10.1038/462041a.

33. Steinman L. Multiple sclerosis: a two-stage disease. Nat Immunol. 2001;2(9): 762-4. https://doi.org/10.1038/ni0901-762

34. Kroenke MA, Carlson TJ, Andjelkovic AV, Segal BM. IL-12- and IL-23modulated T cells induce distinct types of EAE based on histology, CNS chemokine profile, and response to cytokine inhibition. J Exp Med. 2008; 205(7):1535-41. https://doi.org/10.1084/jem.20080159.

35. Stromnes IM, Cerretti LM, Liggitt D, Harris RA, Goverman JM. Differential regulation of central nervous system autoimmunity by $T(H) 1$ and $T(H) 17$ cells. Nat Med. 2008;14(3):337-42. https://doi.org/10.1038/nm1715.
36. Jager A, Dardalhon V, Sobel RA, Bettelli E, Kuchroo VK. Th1, Th17, and Th9 effector cells induce experimental autoimmune encephalomyelitis with different pathological phenotypes. J Immunol. 2009;183(11):7169-77. https://doi.org/10.4049/jimmunol.0901906.

37. Jiang C, Wu B, Xue M, Lin J, Hu Z, Nie X, Cai G: Inflammation accelerates copper-mediated cytotoxicity through induction of six transmembrane epithelial antigens of prostate 4 expression. Immunol Cell Biol 2020.

38. Giacci MK, Bartlett CA, Smith NM, lyer KS, Toomey LM, Jiang H, et al. Oligodendroglia are particularly vulnerable to oxidative damage after neurotrauma in vivo. J Neurosci. 2018;38(29):6491-504. https://doi.org/10.1 523/JNEUROSCI.1898-17.2018.

39. French HM, Reid M, Mamontov P, Simmons RA, Grinspan JB. Oxidative stress disrupts oligodendrocyte maturation. J Neurosci Res. 2009;87(14):3076-87. https://doi.org/10.1002/jnr.22139.

40. Inoue A, Matsumoto I, Tanaka Y, Iwanami K, Kanamori A, Ochiai N, et al. Tumor necrosis factor alpha-induced adipose-related protein expression in experimental arthritis and in rheumatoid arthritis. Arthritis Res Ther. 2009; 11(4):R118. https://doi.org/10.1186/ar2779.

\section{Publisher's Note}

Springer Nature remains neutral with regard to jurisdictional claims in published maps and institutional affiliations.
Ready to submit your research? Choose BMC and benefit from:

- fast, convenient online submission

- thorough peer review by experienced researchers in your field

- rapid publication on acceptance

- support for research data, including large and complex data types

- gold Open Access which fosters wider collaboration and increased citations

- maximum visibility for your research: over $100 \mathrm{M}$ website views per year

At $\mathrm{BMC}$, research is always in progress.

Learn more biomedcentral.com/submissions 